\title{
Role of Dupilumab in Approved Indications of COVID-19 Patient: an Efficacy-Based Nonsystematic Critical Analysis
}

\author{
Pugazhenthan Thangaraju $^{1}$ (D) Nanditha Venkatesan $^{2} \cdot$ T. Y. Sree Sudha ${ }^{1} \cdot$ Sajitha Venkatesan $^{3} \cdot$ Eswaran Thangaraju $^{4}$
}

Accepted: 7 September 2020 / Published online: 10 September 2020

(C) Springer Nature Switzerland AG 2020

\begin{abstract}
With many drugs being tried in the management and treatment of COVID-19, dupilumab is one such monoclonal antibody that has come under the limelight for its possible role as an adjunct therapy in COVID-19 position. There are isolated case reports and series that document a milder course of COVID-19 infection in patients who have already been on dupilumab therapy for treatment of conditions such as atopic dermatitis and chronic rhino-sinusitis with nasal polyp. There is also an ongoing debate regarding the continuation of biologicals in the COVID patient. In this article, a non-systematic critical analysis of dupilumab was performed to delve into this hypothesis further.
\end{abstract}

Keywords Dupilumab $\cdot$ Monoclonal antibody $\cdot$ Chronic rhino-sinusitis with nasal polyp $\cdot$ Atopic dermatitis $\cdot$ COVID-19

\section{Introduction}

Dupilumab is a human monoclonal IgG4 antibody which binds to the alpha subunit of IL-4, 13 receptors and promotes signaling after binding to the IL- $4 \mathrm{R} \alpha$ subunit. While it has already been in use in dermatology clinics for the treatment of atopic dermatitis (AD), it was most recently approved for the indication of chronic rhinosinusitis with nasal polyp (CRSwNP) by the US Food and Drug Association (FDA) on the 26th of June, 2019 [1]. The pathophysiology explored in AD and CRSwNP and the role of the above monoclonal antibodies in link with virus are shown in Fig. 1. A significant anti-viral role of cells, namely, $\mathrm{CD}^{+} \mathrm{T}$ (production of essential specific

This article is part of the Topical Collection on COVID-19

Pugazhenthan Thangaraju

drpugal23@gmail.com

1 Department of Pharmacology, All India Institute of Medical Sciences (AIIMS) Raipur, Raipur, Chhatisgarh, India

2 All India Institute of Medical Sciences (AIIMS) Raipur, Raipur, Chhatisgarh, India

3 Department Of Microbiology, All India Institute of Medical Sciences (AIIMS) Raipur, Raipur, Chhatisgarh, India

4 AKT memorial college of engineering and technology, Kallakurichi, Tamil Nadu, India antibodies) and $\mathrm{CD} 8^{+} \mathrm{T}$ (cytotoxic toward virus infected cells), plays a very important in balancing against the infection of SARS-CoV-2 and associated inflammation. In addition, there is major trigger for differentiation of $\mathrm{T}$ cells into T-helper 1 (Th1) and Th17 in series to the massive release of pro-inflammatory cytokines, namely, tumor necrosis factor $(\operatorname{tnf} \beta)$, interleukin (IL) $(1,6,8$, and 21 ), and monocytes chemoattractant protein-1 (MCP1) by the viral infection. So these lead to the cytokine storm that will prevent the activation of $\mathrm{CD} 8^{+} \mathrm{T}$ cells. The corona virus also seems to stimulate the secretion of IL-4 and IL10 (Th-2 cytokines). This in turn suppresses the inflammation mediated by $\mathrm{T}$ helper cell (1/17). But dupilumab inhibits the function of IL-4 and IL-13. So whether dupilumab usage is beneficial or can lead to increased risk of contraction to SARS-CoV2/complication is still questionable. So at the level of molecular mechanism whether it acts as adjuvant in controlling or against in increased risk should be explored $[2,3]$.

Dupilumab, in the CRSwNP patients, shows its clinical efficacy by minimizing nasal polyp size and increasing quality of life by reducing sinonasal symptoms, particularly in patients with comorbidities such as asthma. Several case reports and letters to the editor have pointed out the safe use of dupilumab in patients in dermatology and otorhinolaryngology outpatient clinics [4].

Dupilumab has been associated with a reduced infection rate in $\mathrm{AD}$ patients. A pooled analysis of seven RCTs 


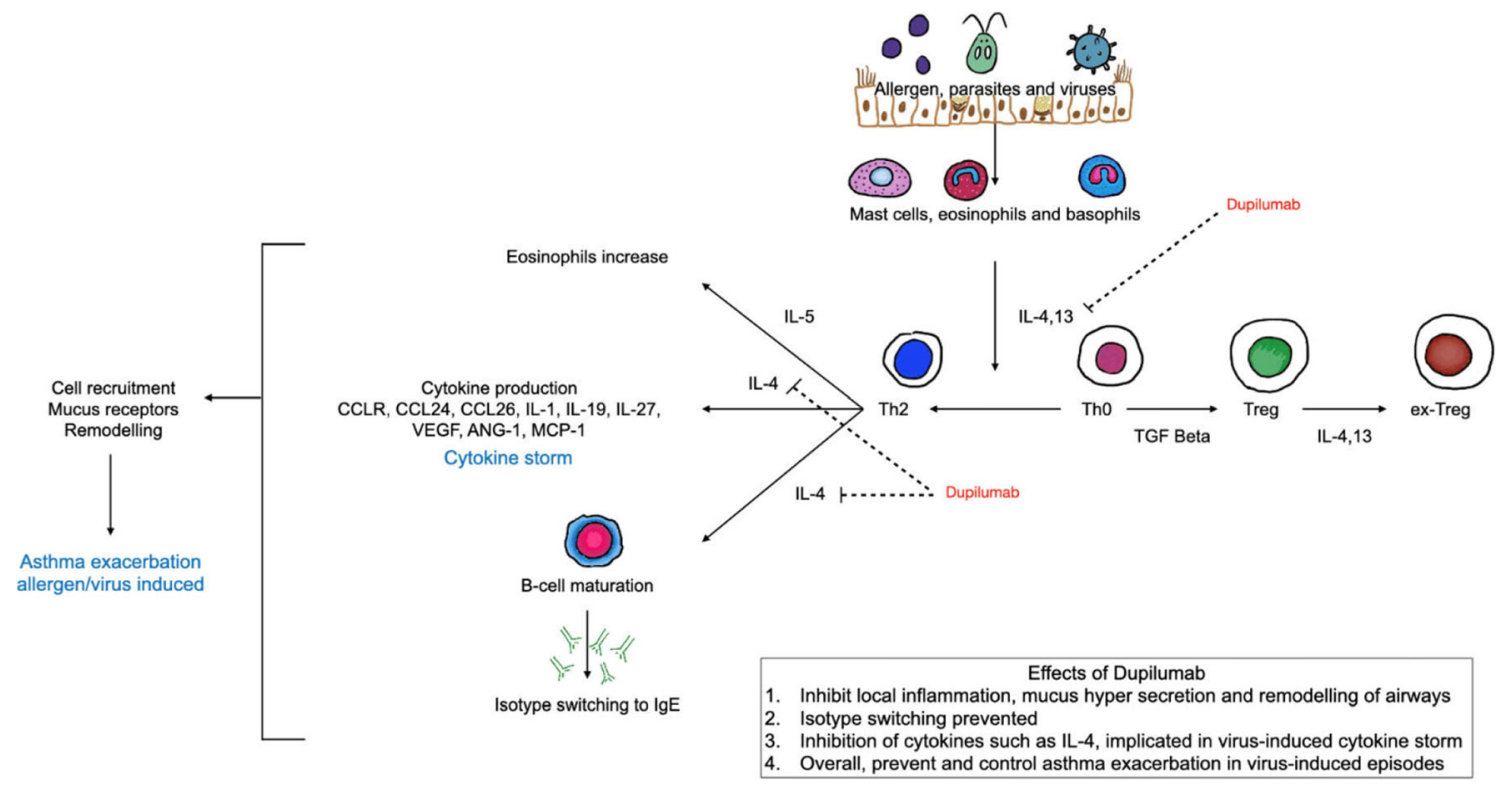

Fig. 1 Pathophysiology in general and adjuvant to virus

on dupilumab-treated AD adults showed a decreased risk of serious infections, skin infections, and herpes infections (eczema herpeticum or herpes zoster) in the dupilumab groups compared with placebo [5]. SOLO 1 and SOLO 2 were RCTs that demonstrated that rates of upper respiratory tract infections were much the same in placebo and dupilumab [6]. There were no reports of more serious lower respiratory infections in dupilumab. A decrease in theoretical risk for COVID-19 in dupilumab-treated patients in addition to its ability to control viral infection-induced asthma has also been noted.

The European Task Force on Atopic Dermatitis recently commented that "Targeted treatment selectively interfering with type-2 inflammation such as dupilumab is not considered to increase the risk for viral infections and might thus be preferred ...in a situation such as COVID-19 pandemic" [7]. However, recommendations swing both ways. The American Academy of Dermatology currently recommends that patients with active COVID-19 infection should discontinue any systemic treatment under the supervision of a dermatologist. The International League of Dermatological Society (ILDS) guidance in contrast asserts that current evidence does not justify the discontinuation of dupilumab therapy for AD in healthy subjects [8].

The current recommendations of the German Society for Allergology and Clinical Immunology for the use of biologics in the treatment of patients with chronic rhinosinusitis and nasal polyp alongside evidence from various published literature point toward the continuation of the treatment [9].

Herein, we explore evidence from published literature and collate their findings that could throw light on the current scenario though a non-systematic critical appraisal.

\section{Methods}

A search was conducted across databases that included PubMed, medrXiv, biorXiv, and SSRN. The search strategy was carried out using keywords such as "Dupilumab," "COVID-19," and similar such terms. We included clinical trials, research studies, case reports, and randomized control trials that employed the use of dupilumab at any point in the treatment of patients who were laboratory confirmed cases of COVID19.

\section{Study Selection}

Given the small number of articles that were found in our search, full-text screening of all articles were done.

\section{Inclusion Criteria}

Patients: Patients, both adult and pediatric population, who are laboratory confirmed cases of COVID-19.

\section{Intervention}

Dupilumab used at any point in the treatment of any disease condition (AD or CRSwNP).

\section{Type of Study}

Clinical trials, research studies, case reports, randomized control trials, and editorials. 


\section{Exclusion Criteria}

- Review articles and comments were not included.

- Dupilumab in patients without lab-confirmed COVID19 infection (Mild flu-like illnesses were not included)

\section{Data Extraction (Selection and Coding)}

Two reviewers independently screened the search results that were documented on Google Sheets. Details that were extracted included author and journal details, year of publication, sample size, age, sex, indication for rational use of dupilumab, duration for treatment, adverse effects observed, and course of COVID-19 infection.

Once this data was retrieved, we measured the total number of COVID19 patients who were on dupilumab and recorded their course of disease.

\section{Statistical Analysis}

The data were extracted in Google Sheets in numbers and converted later to percentages.

\section{Results}

As of July 14, 2020 (Fig. 2), we recorded a total of 15 articles, 11 in PubMed, 4 in the medrXiv/biorXiv COVID-19 preprint collection and 0 relevant searches in the SSRN database. Out of these 15 articles, only 8 met the inclusion criteria. Of these 8 articles, 7 of them used dupilumab for the indication of atopic dermatitis while only 1 article was a case report of a patient on dupilumab for the indication of CRSwNP.

Pooled data from these studies revealed a total of 775 patients on dupilumab, of which 6 developed COVID-19 infection $(0.77 \%$ infection rate). All 6 patients had a mild course of disease and recovered. No deaths were reported in this group. Only 1 out of 8 studies from the non-systematic analysis mentioned the duration of therapy. Out of the 11 results from PubMed, 10 studies recommended continuation of use while 1 review inclined more toward withholding the medication in high-risk groups.

\section{Discussion}

Our results show that the use of dupilumab in general is much lesser for CRSwNP as compared with AD. This could be owing to the difference in time points for when this drug

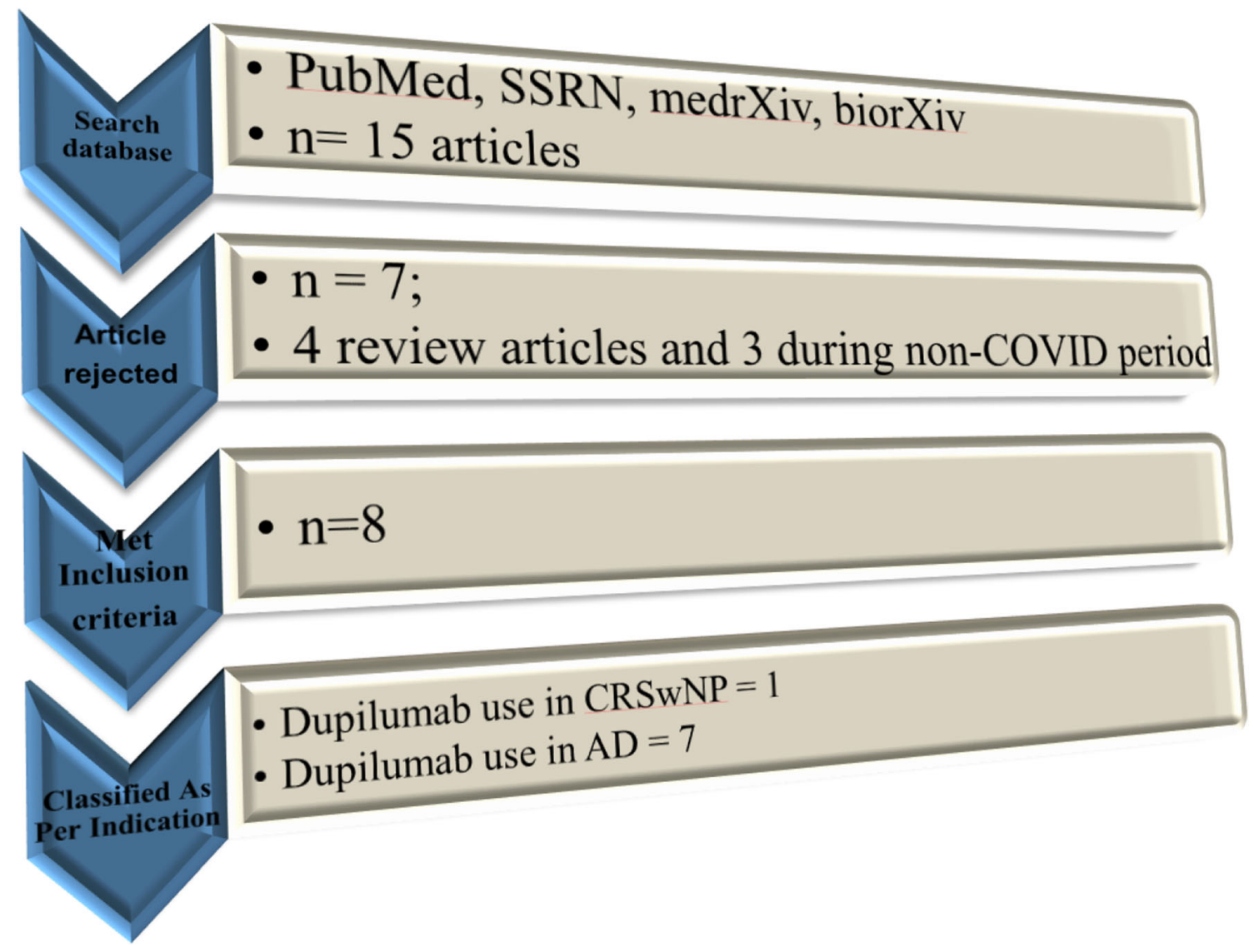

Fig. 2 Flow chart of collected information 
was approved for the two separate indications. Among those patients that developed COVID-19 infection, all of them displayed a mild disease course, tempting us to speculate the possible immune-modulating role of dupilumab.

One case report highlighted the effect of dupilumab in a patient with CRSwNP who developed COVID-19 infection. They hypothesized that the unexpectedly light course of disease could be attributed to (1) reduction in local inflammation and better nasal respiration and sinonasal function, (2) achievement of complete control of asthma in patient in study, and (3) increased eosinophils in patient; strengthening the recently described negative association between an increased number of eosinophils and viral load $[4,10]$.

Dupilumab in CRSwNP treats symptoms such as anosmia, nasal congestion, and cough which are symptoms common to COVID-19. In this case, theoretically, patients on dupilumab might not show classic symptoms of COVID-19. This needs to be borne in mind while these patients are educated about symptoms of COVID-19. It has been cited as a safe choice of medication, which has largely been attributed to its targeted mode of action rather than widespread immunosuppression, latter being a risk factor for severe degree of COVID-19 infection [11]. Abrupt cessation of the therapy in patients could exacerbate previously controlled symptoms, consequently leading to the progression of the inflammation causing a flare up of existing underlying comorbidities that were otherwise under control by the action of dupilumab, such as asthma. With atopic patients having a higher likelihood of developing asthma, viral infections in these patients could exacerbate the condition. In such a context, dupilumab will prove to be effective. Also, it needs to be borne in mind that anti-drug antibodies or neutralizing antibodies could be generated during the period of discontinuation that could eventually render the treatment useless at a later stage [12]. Knowledge of immunological pathways also has shown that the pathways of IL-4 and IL-13, which are Th2 cytokines, have not been implicated in host defense mechanisms against viral infections. However, an excess of the Th2 cytokines (IL-5, 10) have been documented in fatal cases of the 2002 SARS-CoV infection, as compared with recovered patients [13]. With studies showing that heightened immune response and rise in levels of IL4 could potentially worsen the hyperinflammatory response, we speculate that immune-modulation by dupilumab in COVID19 might prove to be beneficial. A recent article also supports on the continuation of monoclonal antibodies when there is no concrete evidence against its usage in atopic dermatitis or chronic rhinosinusitis with nasal polyp [14].

Our study has several limitations that need to be considered while interpreting the results.

- This is a very preliminary collation of existing articles. Very small number of studies have been published in the context of dupilumab use in COVID-19. More evidence in this regard, with a larger sample size, is needed.

- There is lack of any mention of the duration of therapy in these patients. Duration of therapy will influence the effect of the drug on the immune system.

- Many of these patients might have eventually gone on to develop the COVID-19 infection, so the rate of infection is not an absolute indicator.

\section{Conclusion}

Culmination of these effects could push the patient into a more severe form of COVID-19. Flare up for asthma and rhinosinusitis would additionally cause an increase in sputum production and cough, which could increase rates of transmission. Interruption of therapy could lead to the progression of rhinosinusitis, expansion of polyp formation, worsening of comorbidities, and production of anti-drug antibodies.

Unlike other immune-modulatory therapies, clinical trials have shown that the full effect of dupilumab on the immune system occurs much later after initiation. This needs to be borne in mind as duration and initiation of therapy needs more synthesis of evidence. Data regarding duration of therapy and its effect on the immune system also needs to be researched further.

In the context of COVID-19, its role as an immunemodulating agent should be explored further and considered urgently.

\section{Compliance with Ethical Standards}

Conflict of Interest The authors declare that they have no conflict of interest.

Ethical Approval This article does not contain any studies with human participants or animals performed by any of the authors.

Informed Consent Not applicable; This article does not contain any studies with human participants or animals performed by any of the authors.

\section{References}

1. Bachert C, Han JK, Desrosiers M, Hellings PW, Amin N, Lee SE, et al. Efficacy and safety of dupilumab in patients with severe chronic rhinosinusitis with nasal polyps (LIBERTY NP SINUS24 and LIBERTY NP SINUS-52): results from two multicentre, randomised, double-blind, placebo-controlled, parallel-group phase 3 trials. Lancet. 2019;394:1638-50.

2. Li G, Fan Y, Lai Y, Han T, Li Z, Zhou P, et al. Coronavirus infections and immune responses. J Med Virol. 2020;92(4):424 32. 
3. Huang C, Wang Y, Li X, Ren L, Zhao J, Hu Y, et al. Clinical features of patients infected with 2019 novel coronavirus in Wuhan, China. Lancet. 2020;395(10223):497-506.

4. Förster-Ruhrmann U, Szczepek AJ, Claus BH, Heidi OH. COVID19 infection in a patient with severe chronic rhinosinusitis with nasal polyps during therapy with dupilumab. J Allergy Clin Immunol. 2020. https://doi.org/10.1016/j.jaci.2020.05.005.

5. Eichenfield LF, Bieber T, Beck LA, Simpson EL, Thaci D, de Bruin-Weller M, et al. Infections in dupilumab clinical trials in atopic dermatitis: a comprehensive pooled analysis. Am J Clin Dermatol. 2019;20(3):443-56.

6. Simpson EL, Bieber T, Guttman-Yassky E, Beck LA, Blauvelt A, Cork MJ, et al. Two phase 3 trials of dupilumab versus placebo in atopic dermatitis. N Engl J Med. 2016;375(24):2335-48. https:// doi.org/10.1056/NEJMoa1610020.

7. Wollenberg A, Flohr C, Simon D, et al. European Task Force on Atopic Dermatitis (ETFAD) statement on severe acute respiratory syndrome coronavirus 2 (SARS-Cov-2)-infection and atopic dermatitis. J Eur Acad Dermatol Venereol. 2020.

8. International League of Dermatological Societies (ILDS). Guidance on the use of systemic therapy for patients with psoriasis/atopic dermatitis during the COVID-19 (SARS-CoV-2, Coronavirus) pandemic (April2020). https://ilds.org/covid-19/guidance-psoriasisatopic-dermatitis/.

9. DGAKI. Statement of the German Society for Allergology and Clinical immunology (DGAKI) dated March 20, 2020 on the active substances: omalizumab, mepolizumab, reslizumab, benralizumab and dupilumab 2020. Available at: https://gaki.de/wp-content/ uploads/2020/03/Empfehlungen-der-DGAKI-zurBiologikatherapie-und-COVID-19-3-2020.pdf.

10. Liu F, Xu A, Zhang Y, Xuan W, Yan T, Pan K, et al. Patients of COVID-19 may benefit from sustained lopinavir-combined regimen and the increase of eosinophil may predict the outcome of COVID-19 progression. Int J Infect Dis. 2020;95:183-91.

11. Schett G, Sticherling M, Neurath MF. COVID-19: risk for cytokine targeting in chronic inflammatory diseases? Nat Rev Immunol. 2020.

12. Megna M, Napolitano M, Patruno C, Fabbrocini G. Biologics for psoriasis in COVID-19 era: what do we know? [published online ahead of print, 2020 Apr 27]. Dermatol Ther 2020;e13467.

13. Li CK, Wu H, Yan H, Ma S, Wang L, Zhang M, et al. T cell responses to whole SARS coronavirus in humans. J Immunol. 2008;181(8):5490-500.

14. Patruno C, Stingeni L, Fabbrocini G, Hansel K, Napolitano M. Dupilumab and COVID-19: What should we expect? [Published online ahead of print, 2020 May 2]. Dermatol Ther. 2020;e13502. https://doi.org/10.1111/dth.13502.

Publisher's Note Springer Nature remains neutral with regard to jurisdictional claims in published maps and institutional affiliations. 